\title{
Recreo organizado como estrategia para mejorar los niveles actividad física y condición física en adolescentes escolares \\ Organized recess as a strategy to improve physical activity levels and physical condition in adolescents
}

Fernando Rodríguez-Rodríguez*, Sebastián Molina Roblero*, Gerson Luis de Moraes Ferrari**

*Pontificia Universidad Católica de Valparaíso (Chile), **Universidad de Santiago (Chile)

Resumen. El propósito del estudio fue identificar los efectos de una intervención durante recreos escolares sobre la condición física y actividad física en una muestra de adolescentes chilenos. Participaron 50 escolares voluntarios ( 27 chicas), con un promedio de 14,2 $\pm 1,3$ años. Grupo control $(n=21)$ y grupo intervención $(n=29)$. Se realizaron actividades dirigidas en el patio del colegio durante los dos recreos de las mañanas por 12 semanas. Se evaluó el test de $20 \mathrm{~m}$ ida-vuelta, dinamometría manual, composición corporal, cuestionario PAQ-A y acelerometría. El grupo intervención (GI), tuvo mejor nivel de actividad física que el grupo control (GC), durante los recreos, clase de Educación Física, hora de almuerzo y más pasos/día $(\mathrm{p}<0,05)$. En conclusión, la participación activa durante los recreos puede aumentar los minutos de actividad física moderada-vigorosa y mejorar la condición física cardiorrespiratoria.

Palabras Claves: Actividad física, acelerómetría, adolescentes, capacidad aeróbica, recreos.

Abstract. The purpose of this project was to identify the effects of an intervention during school recesses on physical condition and physical activity in Chilean adolescents. Fifty volunteer students (27 girls) participated, with an average of $14.2 \pm 1.3$ years. Control group $(n=21)$ and intervention group $(n=29)$. Targeted activities were carried out in the schoolyard during the two recesses in the morning, for 12 weeks. Pre and post test, the $20 \mathrm{~m}$ shuttle run test, manual dynamometry, body composition, PAQ-A questionnaire and accelerometry were evaluated. The intervention group (GI), had better levels of physical activity than the control group (GC), during recess, physical education class, lunch time and steps/day $(\mathrm{p}<0.05)$. In conclusion, the active participation during recesses can increase minutes of moderate-vigorous physical activity and improve cardiorespiratory fitness.

Keywords: Physical activity, accelerometry, adolescents, aerobic capacity, recess.

\section{Introducción}

El nivel de actividad física (AF), es un factor determinante para la salud de niños y adolescentes. Un bajo nivel de actividad física sostenido en el tiempo, está asociado a factores de riesgo de enfermedades cardiovasculares y metabólicas (Ridgers, Lamb, Timperio, Brown, \& Salmon, 2017), mientras que un alto nivel de AF se asocia a múltiples beneficios para la salud física, mental y cognitiva (Kohl et al., 2013).

La falta de hábitos saludables, la jornada escolar completa en Chile, el tiempo excesivo dentro de las aulas (aproximadamente 6 horas en conductas sedentarias), la utilización de sólo 24 de los 90 minutos semanales de Educación Física (EF) como movimiento real, la falta de profesores especializados, problemas de infraestructura y falta de equipamiento en establecimientos educativos, pueden identificarse como factores del actual panorama en la población escolar, los cuales se acentúan en condiciones de vulnerabilidad (Ministerio del Deporte, 2017).

En Chile, el nivel de AF en escolares adolescentes, está muy lejos de alcanzar las recomendaciones internacionales propuestas por la Organización Mundial de la Salud(OMS), donde 8 de 10 adolescentes no cumple con al menos 60 minutos diarios de AF de moderada-vigorosa intensidad, (Cortínez-O’Ryan \& Aguilar-Farías., 2016). Además, escolares del sector público muestran menores niveles de AF que aquellos de establecimientos privados (Cortinez-O'Ryan \& Aguilar-Farías, 2016).

A pesar de esta realidad, las escuelas se han convertido

Fecha recepción: 26-03-20. Fecha de aceptación: 21-07-20

Fernando Rodríguez-Rodríguez

fernando.rodriguez@pucv.cl en un ambiente ideal para implementar programas y estrategias de AF. Además, las escuelas han surgido como una oportunidad para reducir las conductas sedentarias de niños y adolescentes, ya que pueden modificar los espacios disponibles en los patios de manera efectiva para llamar la atención de los escolares (Groffik, Sigmund, Frömel, Chmelík, \& Nováková-Lokvencová, 2012). No obstante, las intervenciones deben ser apropiadas al contexto y a las necesidades de la comunidad educativa (Rué \& Serrano, 2014). Ante este escenario, los recreos y/o tiempos libres dentro de la jornada escolar, ofrecen una oportunidad valiosa para contribuir a las recomendaciones de $\mathrm{AF}$ en adolescentes, especialmente para los menos activos (Erwin et al., 2012).

La AF en los recreos podría ser una sencilla y económica forma de lograr este objetivo (Shervey \& DiPerna, 2017). Se ha propuesto que durante el recreo, los escolares pueden pasar al menos el 50\% del tiempo realizando actividad física moderada-vigorosa (AFMV) (Stratton \& Mullan, 2005). Sin embargo, se ha indicado que sería más realista alcanzar al menos el 40\% del tiempo en AFMV en recreos (Ridgers, Stratton, \& Fairclough, 2005). En este sentido, un estudio longitudinal español encontró que $<10 \%$ de los escolares cumplieron con las recomendaciones durante el recreo (GraoCruces et al., 2019). Otro estudio observacional realizado en Japón mostró una prevalencia de AFMV de 19,3\% en recreo y tiempo libre (Tanaka et al., 2019). Con estos antecedentes, se hace necesario contribuir con los niveles de AF de adolescentes en la jornada escolar, quebrando la rutina a través de una intervención que modifique los espacios disponibles, que invite a participar a la gran mayoría de adolescentes y que logre generar cambios paulatinos en los niveles de AF de los escolares en los establecimientos educativos. Por esto, el objetivo de este estudio fue examinar los efectos de 
una intervención de recreo organizado, sobre la condición física y AF de una muestra de adolescentes escolares.

\section{Metodos}

\section{Diseño del estudio}

Este estudio, es una intervención educativa cuasi experimental, con una muestra no aleatorizada de adolescentes voluntarios que accedieron a participar del estudio, durante 12 semanas de intevención en los recreos ( $15 \mathrm{~min} \mathrm{c/u}$ ). En la semana 0 y 13 se realizaron las pruebas pre y post test respectivamente y en la semana 13 se aplicó acelerometría durante 7 días incluyendo los 5 dias de jornada escolar (Figura 1).

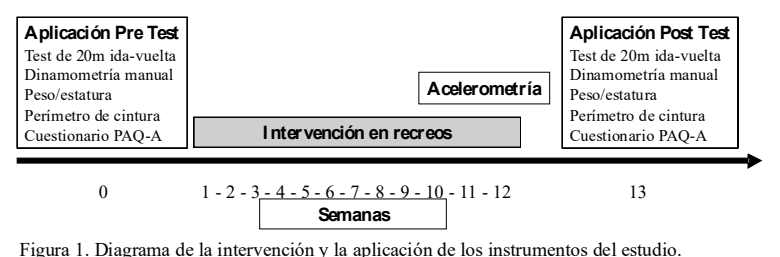

Figura 1. Diagrama de la intervención y la aplicación de los instrumentos del estudio.

\section{Participantes}

Los participantes fueron escolares adolescentes pertenecientes a una escuela pública vulnerable, (índice de vulnerabilidad del 87\%), donde el 75\% correspondieron a escolares prioritarios, con una situación socioeconómica que puede dificultar sus posibilidades de educación (MINEDUC, 2018). Fueron invitados a participar 85 escolares voluntarios pertenecientes a cuatro cursos de primero y segundo grado de escuela secundaria (instituto), de los cuales 35 declinaron participar, quedando un total de 50 adolescentes que se incorporaron al programa (Figura 2). El promedio de edad fue de $14,2 \pm 1,3$ años, $42 \%$ chicas $(n=23)$ y $58 \%$ chicos $(n=27)$, quienes se dividieron en un grupo de intervención (GI) y un grupo control (CG).

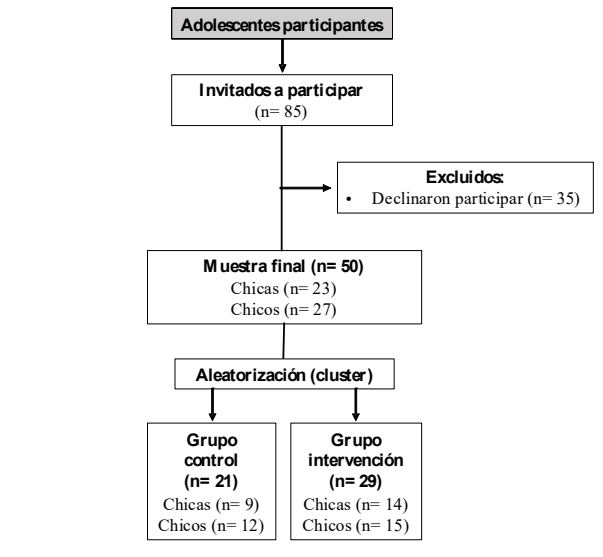

Figura 2. Diagrama de flujo para agrupación de la muestra de participantes.

\section{Evaluación de la condición física}

Se realizaron dos pruebas de la batería ALPHA Fitness (Ruiz et al., 2011), fuerza de prensión manual por dinamometría y Test de ida y vuelta de 20 metros. Se utilizó para esto el dinamómetro JAMAR ${ }^{\circledR}$ Plus + (IL, USA), que proporciona medidas de 0 a $90 \mathrm{~kg}$ y con una precisión de $0,1 \mathrm{~kg}$. Las pruebas de condición física, composición corporal y aplicación de cuestionarios se realizaron en las manañas, una se- mana antes del inicio de la intervención (semana 0). La prueba de dinamometría manual para evaluar la capacidad músculo-esquelética. se realizó con el codo en extensión completa, en una posición de pie, hombros en posición anatómica y brazos paralelos sin contacto con el cuerpo. A cada sujeto se le indicó apretar la manija lo más fuerte posible durante 3 a 5 segundos y realizar dos intentos con cada mano de manera alternada, registrando aquel intento de mayor valor (España-Romero et al., 2010).

También se realizó el test ida y vuelta de 20 metros para la estimación de la capacidad cardiorrespiratoria, por su aceptable validez predictiva y fiabilidad para niños y adolescentes (García \& Secchi, 2014). Una vez completado el test, se utilizó la ecuación de Ruiz et al. (2008) para estimar el $\mathrm{VO}_{2 \mathrm{Max}}$ en adolescentes, la cual considera la última etapa completada, sexo, edad, peso y estatura del participante. Se establecieron categorías de capacidad cardiorrespiratoria según percentiles de la siguiente manera: condición muy mala $\left(<\mathrm{P}_{20}\right)$, condición mala $\left(\mathrm{P}_{20} \mathrm{~d} \gg \mathrm{a}<\mathrm{P}_{40}\right)$, condición media $\left(\mathrm{P}_{40} \mathrm{~d}\right.$ » $\left.\mathrm{a}<\mathrm{P}_{60}\right)$, condición buena $\left(\mathrm{P}_{60} \mathrm{~d}\right.$ » $\left.\mathrm{a}<\mathrm{P}_{80}\right)$ y condición muy buena $\left(\mathrm{e} \gg \mathrm{P}_{80}\right)$ (Ortega et al., 2005).

\section{Composición corporal}

Para medir el peso corporal $(\mathrm{kg})$ se utilizó una balanza digital (Tanita ${ }^{\circledR}$ HD-313, Japan) con precisión de 0,1 kg. Para la evaluación de la estatuta $(\mathrm{cm})$ se utilizó un tallimetro portátil (Rosscraft SRL, Bs As, Argentina). Para el perímetro de cintura $(\mathrm{cm})$ se evaluó utilizando una cinta métrica inextensible (Seca ${ }^{\circledR} 201$, Hamburg, Germany) con una precisión de 1 milímetro (mm).

El peso corporal se evaluó descalso y con la menor ropa posible (short y camiseta). La estatura se evaluó de pie, descalzo y con los pies ligeramente separados y con la menor ropa posible. Se pidió realizar una inspiración profunda y mantenida, mientras el evaluador mantuvo la cabeza en el plano meta obituario, realizando una leve tracción de la cabeza para corregir la compresión cervical. El perímetro de cintura se midió entre el último par de costillas y la cresta ilíaca, al momento del termino de una espiración normal. Posteriormente se calculó el índice de masa corporal $\left(\mathrm{kg} / \mathrm{m}^{2}\right)$ y el riesgo cardiovascular según el perímetro de cintura, acorde a la edad y sexo de los escolares (Ruiz et al., 2011). Todos los escolares fueron evaluados en el primer bloque de clases, en el horario de las 08:30 a las 10:00 horas, previo al desayuno entregado por el misma escuela de manera estándar a todos los participantes.

\section{Evaluación de la Actividad física}

Se aplicó el cuestionario PAQ-A (Physical Activity Questionnaire for Adolescents) para adolescentes entre 13 y 18 años de edad. Este instrumento de autorreporte, valora la actividad física realizada en los últimos 7 días durante el tiempo libre, clases de EF, en diferentes horarios de escuela y el fin de semana. Permite conocer en qué momentos del día y de la semana se es activo. Además, establece una graduación del nivel de actividad física de cada adolescente, con una adecuada fiabilidad y una razonable validez (MartínezGómez et al., 2009). Este instrumento está basado en la validación por acelerometría (de acuerdo al análisis de curva $\mathrm{ROC}=0,68 ; \mathrm{p}<0,001)$, define el valor 2,75 como punto de 
corte entre adolescentes activos e inactivos (Benítez-Porres, Alvero-Cruz, Sardinha, López-Fernández, \& Carneiro, 2016).

Adicionalmente, para estimar los niveles de actividad física durante la semana número 13 de aplicación de la intervención, los escolares portaron acelerómetros triaxiales (Actigraph wGTX3BT, FL, USA). El uso fue en el lado derecho entre la cadera y la cintura, debiendo ser usado durante al menos $10 \mathrm{~h} /$ día, de manera continuada en la jornada escolar. Se validó el rango horario de entre las 8:00 h a 21:00 h y portado por 7 días de uso continuado. Solo podía retirar su uso al momento de ir dormir, al bañarse y cuando realizaran actividades físicas acuáticas.

El acelerómetro se descargó en archivos «.Raw»; que posteriormente se transformaron en archivos «Epoch Data» de 10 segundos para niños (Migueles et al., 2017). Posteriormente se validaron los datos en el software Actilife 6.13.3. Una vez validados todos los archivos se exportaron a una planilla Microsoft Excel para su ordenamiento. La actividad física fue analizada en horarios, comparando los recreos (9:30 a 9:45 h y 11:15 a 11:30 h), la clase de EF y el horario de almuerzo (13:00 a 14:00 h), así como la actividad total de la jornada escolar.

El gasto energético se obtuvo a través de la ecuación de Freedson (Freedson, Melanson, Sirard, 1998). Además, se obtuvo el número de pasos realizados por día, el número de pasos realizados por minuto y los niveles de actividad física expresados en minutos por día, categorizándose la actividad física en los siguientes niveles de intensidad (medido en Metabolic Equivalent of Task - MET): sedentaria $(<1,5$ METs), ligera (1,5-3 METs), moderada ( $>3-6$ METs) y vigorosa ( $>6$ METs) (Cristi-Montero et al., 2014). Para el análisis de acelerómetría, se utilizó el software Actilife Pro. El gasto energético se analizó con la ecuación de Freedson (Freedson et al., 1998) y los METs con la ecuación de Trost en niños (Trost, Loprinzi, Moore, \& Pfeiffer., 2011).

\section{Intervención en recreos}

La intervención se denominó «Desafío Recreo» y tuvo lugar en el patio de la escuela, el cual contó con un espacio deportivo de 14 x 25 metros y un patio general de $700 \mathrm{~m}^{2}$. Se definieron 3 sectores de actividad física organizada: actividades deportivas, actividades de recreación y actividades de acondicionamiento físico, las que se repitieron en varios sectores del establecimiento y se alternaban en cada día de recreo por las 12 semanas de intervención. Se realizaron intervenciones en los dos recreos, los cinco días de la semana. Las actividades fueron dirigidas por el profesor de EF correspondiente a los cursos de secundaria participantes. A este método de participación activa del profesor de EF, le llamaremos «recreo organizado». Se tuvo especial cuidado en que los escolares se mantuvieran activos durante el mayor tiempo posible durante los $15 \mathrm{~min}$ que duraba cada uno de los recreos intervenidos. Cada sector contaba con información grafica relacionada a los beneficios de la AFMV, promoción a la participación en las áreas de actividad física y desafíos prácticos que comprendían realizar actividades durante un tiempo determinado.

\section{Análisis estadístico}

Para el análisis de los datos pre, post test y acelerometría, se utilizó una planilla de registro Microsoft ${ }^{\circledR}$ Excel. El análisis estadístico se realizó con el software SPSS V21 (SPSS Inc., IL, USA). Se comprobó la normalidad de las variables a través de la prueba de Kolmogorov-Smirnov que no arrojó diferencias. No hubo descarte de casos ya que no existieron datos outlier ni hubo deserción al programa. Se calculó la media y desviación estándar de cada resultado de variables contínuas, con el objetivo de describir la condición física de la muestra. Para la comparación de medias de acuerdo al sexo, grupos etarios, GC y GI se utilizó la prueba T-student. Dada la simple comparación de grupos, no se establecieron covariables en el análisis. Para todas las pruebas estadísticas se fijo un nivel de significancia de $\mathrm{p}<0,05$.

\section{Aspectos éticos}

Se aplicó un consentimiento informado y de compromiso, el cual fue firmado por los padres de los jóvenes seleccionados, así como los asentimientos para los escolares. Estos documentos explicaban claramente la metodología a seguir, los objetivos del estudio, los datos requeridos y el compromiso de portar el acelerómetro durante los tiempos establecidos. Además, se explicó la confidencialidad del estudio, su carácter no invasivo y que no conlleva riesgo alguno. Posteriormente se invitó a aquellos que deseaban participar activamente de esta intervención.

Este estudio fue aprobado por el Comité de Bioética correspondiente de la Pontificia Universidad Católica de Valparaíso (CCF02052017) y se siguieron los estandares éticos acordados por la decaración de Helsinki, en la $64^{\mathrm{a}}$ Asamblea General, Fortaleza, Brasil (2013).

\section{Resultados}

En la tabla 1 se muestran los resultados de la evaluación pre y post test del grupo GC, donde no se no observaron cambios importantes en algunas variables a excepción del perímetro de cintura, donde se aprecia una disminución significativa de $2,7 \mathrm{~cm}$ (pre: $78,7 \mathrm{~cm}$ y post: $76 \mathrm{~cm}$; $<<0,05$ ). La fuerza de prensión manual de la mano izquierda disminuyó en $1,8 \mathrm{~kg}$ (pre: $29 \mathrm{~kg}$ y post: $27,2 \mathrm{~kg}$; $\mathrm{p}<0,05$ ) y el peso corporal aumentó significativamente en $1,2 \mathrm{~kg}$ (pre: $62,8 \mathrm{~kg}$ y post: $64 \mathrm{~kg} ; \mathrm{p}<0,05)$ durante el periodo de intervención. A su vez, el GI tuvo cambios significativos en la estatura (pre: $161,4 \mathrm{~cm}$ y post: $162,4 \mathrm{~cm} ; \mathrm{p}<0,05$ ), en la capacidad aeróbica (pre: 39,9 $\mathrm{ml} / \mathrm{kg} / \mathrm{min}^{-1}$ y post: $\left.42,5 \mathrm{ml} / \mathrm{kg} / \mathrm{min}^{-1} ; \mathrm{p}<0,05\right)$, y en el perímetro de cintura (pre: $76,3 \mathrm{~cm}$ y post: $73,5 \mathrm{~cm} ; \mathrm{p}<0,001$ ). No se observaron diferencias significativas en las demás variables.

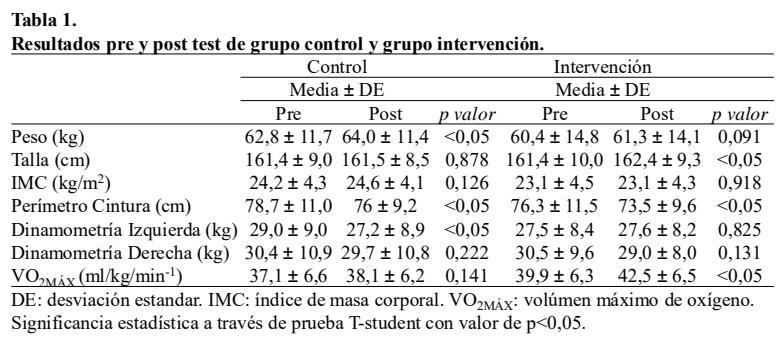

Los resultados del pre y post test del GI por sexo (Tabla 2), evidencian que en chicas sólo hubo cambios importantes en el perímetro de cintura con una disminución de $2,8 \mathrm{~cm}$, (pre: $73 \mathrm{~cm}$ y post: $70,2 \mathrm{~cm} ; \mathrm{p}<0,05$ ), mientras que en el resto 
de las variables no huvo diferencias significativas. Por su parte, los chicos tuvieron una similar disminución en el perímetro de cintura (pre: 79,5 y post: 76,$7 ; \mathrm{p}<0,05$ ). Además, hubo diferencias significativas en la capacidad aeróbica (pre: 39,2 y post: 41,8 , aumento de $2,6 \mathrm{ml} / \mathrm{kg} / \mathrm{min}^{-1} ; \mathrm{p}<0,05$ ).

Tabla 2.

Resultados pre y post test de grupo intervención por género.

\begin{tabular}{|c|c|c|c|c|c|c|}
\hline & \multirow{2}{*}{\multicolumn{2}{|c|}{$\begin{array}{c}\text { Chicas } \\
\text { Media } \pm \text { DE }\end{array}$}} & \multirow{2}{*}{\multicolumn{3}{|c|}{$\begin{array}{c}\text { Chicos } \\
\text { Media } \pm \text { DE }\end{array}$}} & \multirow[b]{3}{*}{ Valor $\mathrm{p}$} \\
\hline & & & & & & \\
\hline & Pre & Post & Valor $\mathbf{p}$ & Pre & Post & \\
\hline Peso (kg) & $54,6 \pm 14,5$ & $55,8 \pm 13,4$ & 0,069 & $66,3 \pm 13,1$ & $66,9 \pm 12,9$ & 0,497 \\
\hline Talla $(\mathrm{cm})$ & $154,9 \pm 7,1$ & $155,9 \pm 5,1$ & 0,230 & $167,3 \pm 8,6$ & $169,0 \pm 7,8$ & 0,052 \\
\hline $\operatorname{IMC}\left(\mathrm{kg} / \mathrm{m}^{2}\right)$ & $22,5 \pm 5,0$ & $22,8 \pm 4,7$ & 0,364 & $23,6 \pm 4,0$ & $23,4 \pm 4,0$ & 0,418 \\
\hline Perímetro Cintura $(\mathrm{cm})$ & $73,0 \pm 11,0$ & $70,2 \pm 8,8$ & $<0,05$ & $79,5 \pm 11,4$ & $76,7 \pm 9,6$ & $<0,05$ \\
\hline Dinamometría Izquierda $(\mathrm{kg}$ ) & $22,4 \pm 5,0$ & $22,0 \pm 4,5$ & 0,445 & $32,6 \pm 8,1$ & $33,3 \pm 7,2$ & 0,449 \\
\hline Dinamometría Derecha $(\mathrm{kg})$ & $25,4 \pm 7,0$ & $24,6 \pm 6,9$ & 0,468 & $35,6 \pm 9,2$ & $33,4 \pm 6,7$ & 0,199 \\
\hline $\mathrm{VO}_{2 \mathrm{MAX}}\left(\mathrm{ml} / \mathrm{kg} / \mathrm{min}^{-1}\right)$ & $40,5 \pm 4,1$ & $43,2 \pm 4,8$ & 0,064 & $39,2 \pm 8,0$ & $41,8 \pm 7,9$ & $<\mathbf{0 , 0 5}$ \\
\hline
\end{tabular}

En la Tabla 3, se puede obervar la conducta activa a través del cuestionario PAQ-A de ambos grupos. En grupo control no presenta cambios significativos, a diferencia del grupo de intervención que mejoró significativamente en «Correr y jugar a la hora de comer», «Activo despues del colegio», «Muy activo el fin de semana» y en el promedio de score total PAQ-A para ambos sexos.

\begin{tabular}{|c|c|c|c|c|c|c|}
\hline \multicolumn{7}{|c|}{$\begin{array}{l}\text { Comparación por sexo del resultado del cuestionario PAQ-A, pre y post intervención } \\
\text { entre grupo control y grupo intervención. }\end{array}$} \\
\hline \multirow[b]{2}{*}{ Chicas } & \multicolumn{3}{|c|}{ Control } & \multicolumn{3}{|c|}{ Intervención } \\
\hline & Pre & Post & pvalor & Pre & Post & p valor \\
\hline Muy actuvo durante educación fisica & 3,6 & 3,8 & 0,366 & 4,1 & 4,1 & 0,403 \\
\hline Correr y jugar a la hora de comer & 1,6 & 1,8 & 0,299 & 2,1 & 2,9 & $<0,05$ \\
\hline Activo despues del colegio & 2,0 & 2,5 & 0,190 & 3,5 & 3,9 & 0,082 \\
\hline Muy activo durante la semana & 2,2 & 2,0 & 0,381 & 3,4 & 3,6 & 0,168 \\
\hline Muy activo el fin de semana & 2,3 & 2,4 & 0,500 & 3,1 & 3,2 & 0,388 \\
\hline $\begin{array}{l}\text { Menor tiempo con poco esfuerzo físico } \\
\text { Chicos }\end{array}$ & 1,8 & 1,8 & 0,500 & 2,9 & 3,2 & 0,082 \\
\hline Muy actuvo durante educación física & 4,1 & 4,1 & 0,500 & 4,4 & 4,6 & 0,217 \\
\hline Correr y jugar a la hora de comer & 2,3 & 2,0 & 0,197 & 2,8 & 2,9 & 0,438 \\
\hline Activo despues del colegio & 3,7 & 3,3 & 0,120 & 2,9 & 3,9 & $<0,05$ \\
\hline Muy activo durante la semana & 2,9 & 2,8 & 0,319 & 2,5 & 2,8 & 0,298 \\
\hline Muy activo el fin de semana & 3,3 & 2,6 & 0,076 & 2,6 & 3,4 & $<0,05$ \\
\hline Menor tiempo con poco esfuerzo físico & 2,2 & 2,6 & 0,120 & 2,6 & 3,0 & 0,157 \\
\hline Score total (chica-chico) PAQ-A & 2,6 & 2,5 & 0,724 & 2,9 & 3,2 & $<0,05$ \\
\hline
\end{tabular}

No obstante, la Figura 3 muestra que la mejora de los niveles globales de actividad física según PAQ-A, es estadísticamente significativa en chicas, pero no en chicos. Incluso el grupo control de chicos, baja su nivel de AF entre pre y post intervención a diferencia de las chicas.

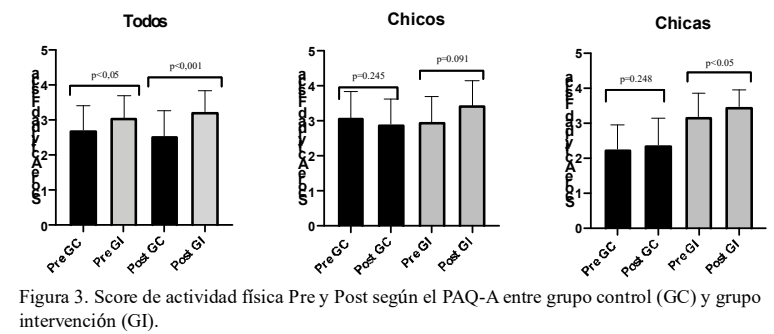

En la Tabla 4 se aprecian las diferencias por sexo en los niveles de AF por acelerometría en la jornada escolar. El GI, tanto en chicos como chicas, mejoran los niveles de AFMV en ambos recreos y en la hora de almuerzo. No obstante, durante la clase de EF, solo mejoraron las chicas. Asimismo, los tiempos sedentarios son menores en GI vs. GC.

\section{Discusión}

El propósito de este estudio fue examinar los efectos de una intervención de recreo organizado, sobre la condición física y AF de una muestra de adolescentes escolares. Además, determinar las diferencias en la composición corporal y en las variables evaluadas de la condición física entre estos grupos. Por último, analizar si existen diferencias por sexo sobre el nivel de AF, composición corporal, capacidad cardiovascular y fuerza de los GI y GC.

\section{Recreos organizados}

Uno de los principales resultados de este estudio, fue la mejora de la condición física cardiorrespiratoria y de los niveles de AF en la jornada escolar de los jóvenes participantes de los recreos organizados (GI).

Al respecto, Verstraete et al., 2006, demuestró que proporcionar equipo de juego en el recreo de la mañana, fue efectivo para aumentar la AFMV de los niños, con una diferencia de $10 \%$ entre GI y GC en el post test (53\% y $43 \%$ en AFMV, respectivamente). Una diferencia similar fue encontrada en la AFMV durante el recreo de la mañana en el presente estudio (GI: 45\% vs GC: 29\%). Otros estudios previos han destacado la efectividad de proporcionar equipo deportivo e implementos en el patio para aumentar los niveles de AF de los escolares durante el recreo (Ridgers, Salmon, Parrish, Stanley, \& Okely, 2012; Parrish, Okely, Stanley, \& Ridgers, 2013).

Otras intervenciones que rediseñaron el ambiente del patio de recreo, usando marcas e implementando infraestructura, mostraron efectos favorables en los niveles de AF durante el recreo (Ridgers, Fairclough, \& Stratton, 2010; Baquet, Aucouturier, Gamelin, \& Berthoin, 2018). No obstante, el tipo de actividad desarrollada por profesores, también podría incrementar el nivel de AF durante el recreo y que puede ser atractivo tanto para niños como para niñas. Esto

Tabla 4.

Comparación de niveles de actividad física por sexo entre los grupos control e intervención $\begin{array}{lllll}\text { durante una jornada escolar. } & & & \\ & \text { Chicas } & \text { Valor } p & \text { Chicos } & \text { Valor } p\end{array}$

\begin{tabular}{|c|c|c|c|c|c|c|}
\hline \multirow{2}{*}{$\mathbf{A F}(\mathbf{m i n})$} & \multicolumn{2}{|c|}{$\begin{array}{c}\text { Chicas } \\
\text { Media } \pm \text { DE }\end{array}$} & \multirow[t]{2}{*}{ Valor $p$} & \multicolumn{2}{|c|}{$\begin{array}{c}\text { Chicos } \\
\text { Media } \pm \text { DE }\end{array}$} & \multirow[t]{2}{*}{ Valor $p$} \\
\hline & Control I & Intervención & & Control 1 & Intervención & \\
\hline \multicolumn{7}{|l|}{$\overline{\text { 1 }^{\text {er }} \text { Recreo }}$} \\
\hline $\begin{array}{l}\text { Tiempo Sedentario } \\
(\mathrm{min} / \text { día })\end{array}$ & $8,8 \pm 2,4$ & $6,3 \pm 1,8$ & $<0,05$ & $8,7 \pm 1,7$ & $6,4 \pm 2,3$ & $<0,05$ \\
\hline AFL (min/día) & $1,7 \pm 0,9$ & $2,1 \pm 0,5$ & 0,157 & $2,2 \pm 0,9$ & $1,9 \pm 0,6$ & 0,209 \\
\hline $\mathrm{AFM}(\mathrm{min} / \mathrm{dia})$ & $4,4 \pm 1,6$ & $5,9 \pm 1,4$ & $<0,05$ & $3,9 \pm 1,2$ & $6,0 \pm 2,1$ & $<0,05$ \\
\hline $\mathrm{AFV}(\mathrm{min} / \mathrm{día})$ & $0,2 \pm 0,2$ & $0,7 \pm 1,0$ & 0,161 & $0,2 \pm 0,2$ & $0,6 \pm 0,6$ & $<0,05$ \\
\hline AFVV (min/día) & $0,0 \pm 0,0$ & $0,2 \pm 0,3$ & 0,070 & $0,01 \pm 0,03$ & $0,1 \pm 0,1$ & $<0,05$ \\
\hline AFMV (min/día) & $4,6 \pm 1,7$ & $6,7 \pm 1,6$ & $<0,05$ & $4,0 \pm 1,2$ & $6,7 \pm 2,2$ & $<0,05$ \\
\hline$\%$ AFMV & $30,7 \pm 11,4$ & $44,5 \pm 10,9$ & $<0,05$ & $26,9 \pm 8,1$ & $44,7 \pm 14,9$ & $<0,05$ \\
\hline Pasos/día & $310 \pm 105$ & $458 \pm 156$ & $<0,05$ & $246 \pm 77$ & $394 \pm 136$ & $<0,05$ \\
\hline \multicolumn{7}{|l|}{$2^{\text {do }}$ Recreo } \\
\hline $\begin{array}{l}\text { Tiempo Sedentario } \\
(\mathrm{min} / \mathrm{dí})\end{array}$ & $10,7 \pm 2,0$ & $7,6 \pm 1,7$ & $<0,05$ & $9,7 \pm 2,4$ & $5,5 \pm 2,8$ & $<0,05$ \\
\hline AFL (min/día) & $1,3 \pm 0,8$ & $2,0 \pm 0,6$ & $<0,05$ & $1,98 \pm 0,9$ & $2,04 \pm 0,7$ & 0,856 \\
\hline $\mathrm{AFM}$ (min/día) & $2,9 \pm 1,3$ & $4,9 \pm 1,4$ & $<0,05$ & $3,1 \pm 1,7$ & $6,2 \pm 2,0$ & $<0,05$ \\
\hline $\mathrm{AFV}(\mathrm{min} / \mathrm{dia})$ & $0,2 \pm 0,2$ & $0,4 \pm 0,3$ & 0,089 & $0,2 \pm 0,2$ & $1,2 \pm 1,4$ & $<0,05$ \\
\hline AFVV (min/día) & $0,0 \pm 0,0$ & $0,2 \pm 0,3$ & 0,103 & $0,0 \pm 0,1$ & $0,2 \pm 0,3$ & 0,061 \\
\hline AFMV (min/día) & $3,0 \pm 1,4$ & $5,4 \pm 1,3$ & $<0,05$ & $3,3 \pm 1,7$ & $7,5 \pm 3,0$ & $<0,05$ \\
\hline$\%$ AFMV & $20,2 \pm 9,5$ & $36,3 \pm 8,7$ & $<0,05$ & $22,1 \pm 11,6$ & $50,1 \pm 19,7$ & $<0,05$ \\
\hline Pasos/día & $204 \pm 102$ & $320 \pm 119$ & $<0,05$ & $180 \pm 89$ & $469 \pm 251$ & $<0,05$ \\
\hline \multicolumn{7}{|l|}{ Tiempo de Almuerzo } \\
\hline $\begin{array}{l}\text { Tiempo Sedentario } \\
\text { (min/día) }\end{array}$ & $31,6 \pm 5,6$ & $25,9 \pm 5,7$ & $<0,05$ & $29,2 \pm 5,7$ & $18,0 \pm 7,4$ & $<0,05$ \\
\hline AFL (min/día) & $4,5 \pm 1,7$ & $6,3 \pm 2,0$ & $<0,05$ & $5,59 \pm 1,9$ & $5,61 \pm 1,7$ & 0,983 \\
\hline $\mathrm{AFM}(\mathrm{min} / \mathrm{dí})$ & $8,2 \pm 4,1$ & $11,9 \pm 4,0$ & $<0,05$ & $9,5 \pm 4,3$ & $18,2 \pm 6,3$ & $<0,05$ \\
\hline $\mathrm{AFV}$ (min/día) & $0,6 \pm 0,4$ & $0,8 \pm 0,4$ & 0,347 & $0,7 \pm 0,7$ & $2,8 \pm 3,2$ & $<0,05$ \\
\hline AFVV (min/día) & $0,03 \pm 0,1$ & $0,2 \pm 0,3$ & 0,108 & $0,1 \pm 0,1$ & $0,3 \pm 0,5$ & 0,061 \\
\hline AFMV (min/día) & $8,8 \pm 4,3$ & $12,8 \pm 4,0$ & $<0,05$ & $10,2 \pm 4,6$ & $21,4 \pm 7,3$ & $<0,05$ \\
\hline$\%$ AFMV & $19,6 \pm 9,4$ & $28,5 \pm 9,0$ & $<0,05$ & $22,6 \pm 10,2$ & $47,5 \pm 16,3$ & $<0,05$ \\
\hline Pasos/día & $524 \pm 229$ & $757 \pm 157$ & $<0,05$ & $513 \pm 235$ & $1216 \pm 530$ & $<0,05$ \\
\hline \multicolumn{7}{|l|}{ Clase de EF } \\
\hline $\begin{array}{l}\text { Tiempo Sedentario } \\
(\mathrm{min} / \mathrm{día})\end{array}$ & $\begin{array}{c}33,4 \pm \\
12,5\end{array}$ & $22,7 \pm 7,0$ & $<0,05$ & $27,1 \pm 10,2$ & $22,9 \pm 6,1$ & 0,196 \\
\hline AFL (min/día) & $6,5 \pm 2,2$ & $8,9 \pm 2,4$ & $<0,05$ & $8,0 \pm 2,1$ & $8,4=$ & 0,651 \\
\hline AFM (min/día) & $\begin{array}{l}17,8 \pm \\
10,5\end{array}$ & $24,9 \pm 7,1$ & 0,068 & $20,5 \pm 7,2$ & $23,6 \pm 5,4$ & 0,211 \\
\hline AFV (min/día) & $1,4 \pm 1,3$ & $3,0 \pm 2,3$ & 0,089 & $3,9 \pm 4,7$ & $4,1 \pm 1,8$ & 0,892 \\
\hline AFVV (min/día) & $0,8 \pm 1,7$ & $0,6 \pm 1,0$ & 0,657 & $0,5 \pm 0,5$ & $1,0 \pm 1,1$ & 0,150 \\
\hline AFMV (min/dia) & $20,1 \pm 11,2$ & $28,4 \pm 7,1$ & $<0,05$ & $24,9 \pm 9,6$ & $28,7 \pm 5,4$ & 0,202 \\
\hline$\%$ AFMV & $\begin{array}{c}33,5 \pm \\
18,7\end{array}$ & $47,3 \pm 11,8$ & $<0,05$ & $41,5 \pm 16,0$ & $47,9 \pm 9,0$ & 0,202 \\
\hline Pasos/día & $1156 \pm 612$ & $1770 \pm 607$ & & $1446 \pm 782$ & & 0,381 \\
\hline \multirow{2}{*}{\multicolumn{7}{|c|}{$\begin{array}{l}\mathrm{AF}=\text { Actividad Física; } \mathrm{AFL}=\text { Actividad Física Ligera; } \mathrm{AFM}=\text { Actividad Física Moderada; } \\
\mathrm{AFV}=\text { Actividad Física Vigorosa; } \mathrm{AFVV}=\text { Actividad Física Muy Vigorosa; AFMV= Actividad }\end{array}$}} \\
\hline & & & & & & \\
\hline & & & & & & \\
\hline
\end{tabular}


es importante de considerar, ya que que el recreo no genera automaticamente AF (Sallis et al., 2001). Por lo tanto, se hace necesario implemetar actividades durante el recreo (Erwin, Beighle, Carson, \& Castelli, 2013), donde la participación del profesor es fundamental. Cuando el profesor de EF provee supervisión y seguridad a la actividad, se denomina como recreo supervisado y cuando el profesor supervisa, da seguridad y entrega instrucciones, se denomina como recreo organizado (Coolkens, Ward, Seghers, \& Iserbyt, 2019). En este estudio se ha trabajado bajo esta metodología de recreo organizado, que permitió hacer una importante diferencia con los escolares del grupo control especialmente en las chicas quienes tuvieron un mayor porcentaje de tiempo en AFMV (33,5\% vs. 47,3\%; $<<0,05)$. Al respecto, el estudio de Coolkens et al. (2019), definió un menor tiempo sedentario (24\% vs. $30 \%$ ) y un mayor tiempo de AFMV (76\% vs. $70 \%$ ) del GI al realizar recreo organizado, vs. el recreo supervisado, respectivamente.

Además, los niños son más activos físicamente en los recreos cuando tienen acceso a espacios adecuados, equipamiento y cuando los adultos organizan juegos y actividades durante el recreo (Bleeker, Beyler, James Burdumy, \& Fortson, 2015). No obstante, esta estrategia tiene un costo mayor de recurso humano, a diferencia de poner a disposición solo materiales y espacio físico, como en el recreo supervisado.

Otro estudio evaluó la relación costo-efectividad de un programa de intervención de 2 años, donde el mismo personal escolar dedicó 15 minutos adicionales a actividades relacionadas con el recreo, estimando un costo de 33 centavos de dolar/hora/MET, pero al mismo tiempo la AF incrementó en 1,8 MET/hora/día por cada estudiante (Wang, Siahpush, Chen, \& Huberty, 2017). En el presente estudio hubo similar dedicación del profesor a cargo y con mejoras positivas sobre la AF. Aún así, cada establecimiento educativo puede buscar la mejor manera de aumentar los niveles de AF en los recreos, aunque la combinación de varias estrategias parece ser la más efectiva (D’Haese, Van Dyck, De Bourdeaudhuij, \& Cardon, 2013).

\section{Efectos de la intervención en recreos sobre el nivel de actividad física escolar}

Varios estudios han evaluado el nivel de AF durante la jornada escolar usando acelerómetros. Un estudio realizado en adolescentes españoles ( $\mathrm{n}=231 ; 14,6 \pm 1,2$ años), donde se compararon los niveles de AFMV durante la jornada escolar (clase EF, recreos y tiempo extra-curricular). evidenció que sólo un 10,8\% de la AFMV se logró durante los recreos y sólo un $26,7 \%$ de AFMV en clases de EF, siendo los chicos más activos que las chicas en todos los contextos $(\mathrm{p}<0,001)$. Además en el grupo de mayor edad (14,4-15,5 años) fueron más activos durante las clases de EF que el grupo de menor edad, y este último grupo (12,5-13,5 años) fue más activo que el grupo de mayor edad durante los recreos $(\mathrm{p}<0,001)$ (Viciana, Mayorga-Vega, \& Martínez-Baena, 2016). Otro estudio realizado en escolares chilenos ( $\mathrm{n}=89 ; 13,4 \pm 0,7$ años), mostró un porcentaje de AFMV en clases de EF superior al del tiempo extra-curricular y recreos ( $\mathrm{p}<0,001)$, en el que sólo el $6,9 \%$ del tiempo en recreos se realizó en AFMV y en el que los chicos fueron más activos que las chicas en todos los contextos
( $<<0,05)$, (Mayorga-Vega, Saldías, \& Viciana, 2017). Similares resultados fueron encontrados en el presente estudio.

Otro reciente estudio chileno que evaluó el efecto de una intervención de 8 meses durantes clases de $60 \mathrm{~min}$, encontró también mayor AFMV en clases de EF que en recreos. No obstante, la AF en los recreos fue menor en el GI probablemente porque ya recibieron intervención durante las clases, provocando un efecto compensatorio (disminución) en recreos (Rodríguez-Rodríguez, Cristi-Montero \& Castro-Piñero, 2020).

Dentro de las recomendaciones de AF durante la jornada escolar, tanto niños como adolescentes, deben gastar al menos un 40\% del tiempo en AFMV durante los recreos (Ridgers et al., 2005), y que al menos participen el 50\% del tiempo de una clase de EF en AFMV (U.S. Department of Health and Human Services, 2010; Stratton \& Mullan, 2005). De acuerdo a esto, este estudio revela que el GI alcanzó un $44 \%$ del tiempo en AFMV durante los recreos, en comparación a un $24,9 \%$ alcanzado por el $\mathrm{GC}(\mathrm{p}<0,05)$. En relación al tiempo de AFMV durante los diversos períodos escolares, ocurre lo mismo al observar la clase de EF (GI: 47,6\% vs. GC: $38,1 \%$ ), el tiempo de almuerzo (GI: $38,3 \%$ vs. GC: $21,4 \%$ ) y los pasos/día(GI: 3.517 pasos vs. GC: 2.305 pasos) siendo significativas las diferencias en todos los casos $(p<0,05)$.

En relación al tiempo libre (tiempo de almuerzo + recreos), este puede contribuir entre el $30 \%$ y $40 \%$ de la AFMV recomendada por día (Ridgers et al., 2017). A partir de esta información, podemos establecer que el GI logra un 44\% deAFMV durante los recreos, cumpliendo con el porcentaje recomendado. Ambos grupos no alcanzan el 50\% de AFMV durante las clases de EF y sólo el GI supera las recomendaciones de AFMV durante el tiempo libre con un 50,8\% en comparación con el $28,5 \%$ del GC.

Las sugerencias de pasos/día indican que, los niños deberían alcanzar entre 3.474-7.594 pasos y 2.900-6.070 para niñas durante una jornada escolar (Brusseau et al., 2011). Los participantes del GI, tanto chicos como chicas, cumplen con la sugerencia de pasos durante la jornada escolar con 3.715 pasos/días y 3.305 pasos/día, respectivamente.

En el presente estudio se puede observar una importante diferencia por sexo, donde las chicas del GI vs. GC, alcanzan un $40,4 \%$ y $25,5 \%$ de AFMV durante los recreos respectivamente $(\mathrm{p}<0,05)$. En comparación con el $1^{\mathrm{er}}$ recreo, existe una disminución en los minutos de AFMV durante el $2^{\text {do }}$ recreo en ambos grupos. No obstante, la mayor diferencia entre chicas se presenta en este último periodo $(\mathrm{p}<0,05)$. Existen diferencias significativas durante la clase de $\operatorname{EF}(p<0,05)$, entre ambos grupos de chicas, lo que da cuenta de la importancia que tiene la participación de las chicas en el nivel total de AFMV para el GI.

Por su parte, los chicos del GI y del GC, alcanzan un $47,4 \%$ vs. $24,5 \%$ de AFMV durante los recreos $(p<0,05)$, en donde el nivel de AFMV es menor en el GC y mayor en el GI durante el $2^{\mathrm{do}}$ recreo en comparación con el $1^{\mathrm{er}}$ recreo. Inclusive el GI alcanza el 50\% del tiempo en AFMV en el $2^{\text {do }}$ recreo, logrando la mayor diferencia entre el GC vs. GI en chicos $(p<0,05)$. Sin embargo, en la clase de EF no se observan diferencias en los niveles de AF entre los adolescentes chicos del GI vs. GC ( $p=0,202)$. Estos resultados son muy prometedores y evidencian un alto cumplimiento de las reco- 
mendaciones de AFMV. Al respecto, una reciente revisión sistemática determinó que los adolescentes solo gastan entre 3\%-8\% del tiempo en AFMV durante la jornada escolar sin intervención (Grao-Cruces, Velásquez-Romero, \& Rodriguez-Rodríguez, 2020).

Por otra parte, los resultados del cuestionario PAQ-A, nos muestran una diferencia significativa en el score total de $\mathrm{AF}$, entre el GI y el GC, en pre-post test $(\mathrm{p}<0,05 ; \mathrm{p}=0,724$, respectivamente). Esta diferencia se da especialmente en la $\mathrm{AF}$ a la hora de almuerzo (chicas) y en la AF en el tiempo extraescolar (chicos). El score total alcanzado en promedio por el GC posterior a la intervención fue de 2,5 y de 3,2 para el GI. Este último superó el punto de corte definido para determinar el cumplimiento de los $60 \mathrm{~min}$ de AFMV/día, estalecido en $>2,75$ (Benítez-Porres et al., 2016). Sin embargo, es necesario tener en cuenta que, el PAQ-A es un cuestionario que valora el nivel de AF durante los últimos 7 días de la semana y no exclusivamente de los días de escolaridad, por lo que este aumento en el nivel de AF, no se puede asociar exclusivamente de la intervención realizada durante la jornada escolar.

Efectos de la intervención en recreos sobre la condición física

Los resultados de este estudio muestran que existen diferencias pre y post intervención en ambos grupos, donde se observa que en el GC aumentó el peso corporal, y disminuyó el perímetro de cintura y la fuerza por dinamometría, a diferencia del GI que aumentó la estatura y la condición física cardiorrespiratoria y disminuyó el perímetro de cintura. Resultados similares han sido encontrados en escolares chilenos (12-15 años), donde las chicas tuvieron un perímetro de cintura de $72 \mathrm{~cm}$ y de $74 \mathrm{~cm}$ en el caso de los chicos (Floody et al., 2015). Por otro lado, según los resultados del SIMCE-EF 2012 (Sistema de medición de la calidad de la educación física), escolares de 13 a 15 años, obtubieron un promedio de $72,7 \mathrm{~cm}$ y $74,7 \mathrm{~cm}$, para chicas y chicos respectivamente (Garcia-Rubio et al., 2015). Esto demuestra un promedio de perímetro de cintura similar a los encontrados en esta intervención (12-16 años).

En relación a la condición física cardiorrespiratoria, un estudio anterior encontró que chicas y chicos lograron 41,2 y $39,9 \mathrm{ml} / \mathrm{kg} / \mathrm{min}^{-1}$ respectivamente (Ibarra et al., 2017). Similares resultados se encorntraron en el estudio colombiano FUPRECOL (Prieto-Benavides et al., 2015), revelando que los participantes del GI tuvieron mejores indicadores en la capacidad aeróbica post intervención. Los puntos de corte propuestos por el grupo FITNESSGRAM del Cooper Institute, definen que el umbral de salud cardiovascular en adolescentes chicos estaría fijado en $42 \mathrm{ml} / \mathrm{kg} / \mathrm{min}^{-1}$ y para chicas, a partir de los 14 años, en $35 \mathrm{ml} / \mathrm{kg} / \mathrm{min}^{-1}$ y $38 \mathrm{ml} / \mathrm{kg}$ / $\mathrm{min}^{-1}$ para edades inferiores (Ortega et al., 2005). Al comparar estos valores base con los resultados de esta intervención, se observa que los escolares que participaron en el GI, cumplen con las recomendaciones para condición física cardiorrespiratoria.

\section{Fortalezas y Limitaciones}

La principal fortaleza de este estudio ha sido la utilización de acelerómetros como método objetivo para estima- ción los niveles de AF durante la jornada escolar.

Dentro de las limitaciones se destaca el pequeño y local tamaño de la muestra que no permite transferir los resultados de manera representativa. Al mismo tiempo el cuestionario usado tiene sus limitaciones de objetividad en la estimación de la actividad física. Además, las dificultades temporales y de disponibilidad de materiales impidió utilizar los acelerometros en el pre-test que permitiera definir de manera más objetiva los cambios producidos por la intervención sobre la AF.

\section{Conclusiones}

En base al objetivo general propuesto, la intervención ha tenido efectos positivos en la AF de los participantes del GI en recreos organizados. Los resultados obtenidos, demuestran que la participación activa durante los recreos puede contribuir a aumentar los minutos de AFMV recomendados por día y disminuir los tiempos sedentarios durante un día escolar. Si bien, la participación activa durante los recreos, podría tener relación con el nivel de AFMV durante las clases de EF, específicamente en chicas, es necesario seguir investigando esta relación con mayor profundidad. No obstante, los niveles de AFMV recomendados para la clase de EF no se cumplen. Además, los cambios positivos en la condición física, especialmente en la condición física cardiorrespiratoria y el perímetro de cintura, respaldan la realización de este tipo de programas de AF en contextos escolares y su aporte a la salud.

Por lo tanto, se vuelve necesario seguir realizando este tipo de investigaciones en contextos escolares, con instrumentos de medición objetivos y en una muestra representativa a nivel regional o nacional, que permita demostrar los efectos positivos que tienen los recreos sobre la AF y la salud de los escolares.

\section{Referencias}

Baquet, G., Aucouturier, J., Gamelin, F. X., \& Berthoin, S. (2018). Longitudinal follow-up of physical activity during school recess: impact of playground markings. Frontiers in public health, 6. https://doi.org/10.3389/fpubh.2018.00283

Benítez-Porres, J., Alvero-Cruz, J. R., Sardinha, L. B., LópezFernández, I., \& Carneiro, E.A. (2016). Cut-off values for classifying active children and adolescents using the Physical Activity Questionnaire: PAQ-C and PAQ-A. Nutricion Hospitalaria, 33(5), 1036-1044. https://doi.org/ http://dx.doi.org/10.20960/nh.574

Bleeker, M., Beyler, N., James Burdumy, S., \& Fortson, J. (2015). The impact of playworks on boys' and girls' physical activity during recess. Journal of school health, 85(3), 171-178. https://doi.org/10.1111/josh.12235

Brusseau, T. A., Kulinna, P. H., Tudor-Locke, C., Ferry, M., van der Mars, H., \& Darst, P. W. (2011). PedometerDetermined Segmented Physical Activity Patterns of Fourth- and Fifth-Grade Children. Journal of Physical Activity and Health, 8(2), 279-286. https://doi.org/ 10.1123/jpah.8.2.279

Coolkens R., Ward P., Seghers J., Iserbyt P. (2018). The Effect of Organized Versus Supervised Recess on Elementary 
School Children's Participation, Physical Activity, Play, and Social Behavior: A Cluster Randomized Controlled Trial. Journal of Physical Activity \& Health, 15 (10), 747754. https://doi.org/10.1123/jpah.2017-0591

Cortinez-O'ryan, A., \& Aguilar-Farias, N. (2016). Reporte de notas chileno sobre la actividad fisica en niños y adoloescentes 2016. Retrieved from www.flaticon.com

Cristi-Montero, C., \& Rodríguez, F. R. (2014). The paradox of being physically active but sedentary or sedentary but physically active. Revista Médica de Chile, 142(1), 7278. https://doi.org/10.4067/S0034-98872014000100011

D’Haese, S., Van Dyck, D., De Bourdeaudhuij, I., \& Cardon, G. (2013). Effectiveness and feasibility of lowering playground density during recess to promote physical activity and decrease sedentary time at primary school. BMC public health, 13(1), 1154. https://doi.org/10.1186/ 1471-2458-13-1154

Erwin H, Beighle A, Carson RL, Castelli DM (2013). Comprehensive school-based physical activity promotion: a review. Quest. 65(4):412-428. doi:10.1080/ 00336297.2013.791872

Erwin, H., Abel, M., Beighle, A., Noland, M. P., Worley, B., \& Riggs, R. (2012). The Contribution of Recess to Children's School-Day Physical Activity. Journal of Physical Activity and Health, 9(3), 442-448. https://doi.org/10.1123/ jpah.9.3.442

España-Romero, V.,Artero, E. G., Jimenez-Pavón, D., CuencaGarcia, M., Ortega, F. B., Castro-Piñero, J., Sjöstrom, M., Castillo-Garzón, M.J., Ruiz, J. R. (2010). Assessing healthrelated fitness tests in the school setting: Reliability, feasibility and safety; The ALPHA study. International Journal of Sports Medicine, 31(7), 490-497. https:// doi.org/10.1055/s-0030-1251990

Floody, P. D., Navarrete, F. C., Guzmán, I. P. G., Mayorga, D. J., Ramírez-Campillo, R., Jara, C. C., Lagos, G., Inostroza, H. D. (2015). Levels of obesity, fasting glycemia and physical condition in chilean students. Nutricion Hospitalaria, 31(6), 2445-2450. https://doi.org/10.3305/ nh.2015.31.6.8932

Freedson P.S., Melanson E., Sirard J. (1998). Calibration of the Computer Science and Applications, Inc. accelerometer. Medicine and science in sports and exercise. 30(5):777-81. https://doi.org/10.1097/00005768199805000-00021

García, G. C., \& Secchi, J. D. (2014). Test course navette de 20 metros con etapas de un minuto. Una idea original que perdura hace 30 años. Apunts Medicina de l'Esport, 49(183), 93-103. https://doi.org/10.1016/ j.apunts.2014.06.001

Garcia-Rubio, J., Lopez-Legarrea, P., Gomez-Campos, R., Cossio-Bolaños, M., Merellano-Navarro, E., \& Olivares, P. R. (2015). Ratio cintura-Estatura y riesgo de síndrome metabólico en adolescentes chilenos. Nutricion Hospitalaria, 31(4), 1589-1596. https://doi.org/10.3305/ nh.2015.31.4.8535

Grao-Cruces, A., Velásquez-Romero, M. J., \& RodriguezRodríguez, F. (2020). Levels of Physical Activity during School Hours in Children and Adolescents: A Systematic Review. International Journal of Environmental Research and Public Health, 17(13), 4773. https://doi.org/10.3390/ ijerph17134773

Grao-Cruces, A.; Sánchez-Oliva, D.; Segura-Jiménez, V.; Cabanas-Sánchez, V.; Martínez-Gómez, D.; RodríguezRodríguez, F.; Moreno, L.A.; Castro-Piñero, J. (2019). Changes in compliance with schoolbased physical activity recommendations in Spanish youth: The UP\&DOWN longitudinal study. Scand. J. Med. Sci. Sports, 29, 554-565. https://doi.org/10.1111/sms.13355

Groffik, D., Sigmund, E., Frömel, K., Chmelík, F., \& Nováková Lokvencová, P. (2012). The contribution of school breaks to the all-day physical activity of 9- and 10-year-old overweight and non-overweight children. International Journal of Public Health, 57(4), 711-718. https://doi.org/ $10.1007 / \mathrm{s} 00038-012-0355-\mathrm{z}$

Ibarra Mora, J., Hernández-Mosqueira, C., Hermosilla Palma, F., PavezAdasme, G., \& Martinez Salazar, C. (2017). Estado nutricional y desempeño físico de una muestra de escolares de 14 y 15 años de la ciudad de Chillán, Chile. Revista Española de Nutrición Humana y Dietética, 21(3), 248. https://doi.org/10.14306/renhyd.21.3.363

Kohl, H. W., Cook, H. D., Van Dusen, D. P., Kelder, S. H., Kohl, H. W., Ranjit, N., \& Perry, C. L. (2013). Educating the study body: taking physical activity and physical education to school. Chapter 4: Physical Activity, Fitness, and Physical Education: Effects on Academic Performance. Journal of School Health (Vol. 81). https://doi.org/ $10.17226 / 18314$

Martínez-Gómez, D., Martínez-De-Haro, V., Pozo, T., Welk, G. J., Villagra,A., Calle, M. E., Marcos, A., Veiga, O. L. (2009). Fiabilidad y validez del cuestionario de actividad física paq-a en adolescentes españoles. Revista Espanola de Salud Publica, 83(3), 427-439. https://doi.org/10.1590/ S1135-57272009000300008

Mayorga-Vega, D., Saldías, M. P., \& Viciana, J. (2017). Comparison of Moderate-To-Vigorous Physical Activity Levels Between Physical Education, School Recess and After-School Time in Secondary School Students: an Accelerometer-Based Study. Kinesiology, 49(2), 242-251. https://doi.org/10.26582/k.49.2.1

Migueles, J. H., Cadenas-Sanchez, C., Ekelund, U., Nyström, C. D., Mora-Gonzalez, J., Löf, M., Labayen I., Ruiz J. R \& Ortega, F. B. (2017). Accelerometer data collection and processing criteria to assess physical activity and other outcomes: a systematic review and practical considerations. Sports medicine, 47(9), 1821-1845.

MINEDUC. (2018). Alumnos prioritarios y preferentes, 20162018. https://sep.mineduc.cl/alumnos-prioritarios-preferente/

Ministerio del Deporte. (2017). Plan Estratégico Nacional de Actividad Física y Deporte 2016-2025 Tabla de contenidos, 0-61. Retrieved from http://www.mindep.cl/wpcontent/uploads/2016/06/Plan-Estratégico-NacionalMindep-Resumen.pdf

Ortega, F. B., Ruiz, J. R., Castillo, M. J., Moreno, L.A., GonzálezGross, M., Wärnberg, J., ... Blay, M. G. (2005). Bajo nivel de forma física en los adolescentes españoles. Importancia para la salud cardiovascular futura (Estudio AVENA). Revista Espanola de Cardiologia, 58(8), 898-909. https:// doi.org/10.1157/13078126

Parrish, A. M., Okely, A. D., Stanley, R. M., \& Ridgers, N. D. 
(2013). The effect of school recess interventions on physical activity. Sports medicine, 43(4), 287-299. https:/ /doi.org/10.1007/s40279-013-0024-2

Prieto-Benavides, D. H., Correa-Bautista, J. E., \& RamírezVélez, R. (2015). Niveles de actividad física, condición física y tiempo en pantallas en escolares de Bogotá, Colombia: Estudio FUPRECOL. Nutricion Hospitalaria, 32(5), 2184-2192. https://doi.org/10.3305/nh.2015.32.5.9576

Ridgers N.D., Fairclough S.J., Stratton G. (2010). Twelve-month effects of a playground intervention on children's morning and lunchtime recess physical activity levels. J Phy Act Health. 7:167-75. https://doi.org/10.1123/ jpah.7.2.167

Ridgers, N. D., Lamb, K. E., Timperio, A., Brown, H., \& Salmon, J. (2017). Investigating Children's Short-Term Responses to Imposed or Restricted Physical Activity. Journal of Physical Activity and Health, 1-8. https://doi.org/10.1123/ jpah.2017-0297

Ridgers, N. D., Salmon, J., Parrish, A. M., Stanley, R. M., \& Okely,A. D. (2012). Physical activity during school recess: a systematic review. American journal of preventive medicine, 43(3), 320-328. https://doi.org/10.1016/ j.amepre.2012.05.019

Ridgers, N. D., Stratton, G., \& Fairclough, S. J. (2005). Assessing physical activity during recess using accelerometry. Preventive Medicine, 41(1), 102-107. https://doi.org/10.1016/j.ypmed.2004.10.023

Rodríguez-Rodríguez, F., Cristi-Montero, C., \& Castro-Piñero, J. (2020). Physical Activity Levels of Chilean Children in a National School Intervention Programme. A Quasi-Experimental Study. International Journal of Environmental Research and Public Health, 17(12), 4529. https://doi.org/ 10.3390/ijerph17124529

Rué Rosell, L., \& Serrano Alfonso, M. Á. (2014). Educación física y promoción de la salud: estrategias de intervención en la escuela. Retos. Nuevas Tendencias En Educación Fisica, Deporte y Recreación., 25, 186-191. Retrieved from http://diposit.ub.edu/dspace/handle/2445/49164

Ruiz, J. R., Ortega, F. B., Martínez-Gómez, D., Labayen, I., Moreno, L.A., De Bourdeaudhuij, I., Manios, Y., GonzalezGross, M., Mauro, B., Molnar, D., Widhalm, K., Marcos, A., Beghin, L., Castillo, M.J., Sjöström, M. (2011). Objectively measured physical activity and sedentary time in european adolescents. American Journal of Epidemiology, 174(2), 173-184. https://doi.org/10.1093/ aje/kwr068

Ruiz, J. R., Ramirez-Lechuga, J., Ortega, F. B., Castro-Piñero, J., Benitez, J. M., Arauzo-Azofra, A., Sánchez, C., Sjöström, M., Castillo, M.J., Gutierrez, A., Zabala, M. (2008). Artificial neural network-based equation for estimating VO2maxfrom the $20 \mathrm{~m}$ shuttle run test in adolescents. Artificial Intelligence in Medicine, 44(3), 233-245. https:/ /doi.org/10.1016/j.artmed.2008.06.004

Sallis J.F., Conway T.L., Prochaska J.J., McKenzie T.L., Marshall S.J., Brown M. (2001). The association of school environments with youth physical activity. Am J Public Health. 91(4):618-620.PubMed ID: 11291375 doi:10.2105/ AJPH.91.4.618

Shervey, S. W., \& DiPerna, J. C. (2017). Engagement in Physical Activity During Recess: Gender and Grade Level
Differences in the Elementary Grades. Journal of Physical Activity and Health, 14(9), 677-683. https://doi.org/ 10.1123/jpah.2014-0499

Stratton G., Mullan E. (2005). The effect of multicolor playground markings on children's physical activity level during recess. Prev Med. 41(5-6):828-833. PubMed ID: 16137756 doi:10.1016/j.ypmed.2005.07.009

Tanaka, C., Tanaka, M., Inoue, S., Okuda, M., Tanaka, S. (2019). Gender differences in physical activity and sedentary behavior of Japanese primary school children during school cleaning time, morning recess and lunch recess. BMC Public Health, 19, 985. https://doi.org/10.1186/ s12889-019-7256-5

Trost, S. G., Loprinzi, P. D., Moore, R., \& Pfeiffer, K.A. (2011). Comparison of accelerometer cut points for predicting activity intensity in youth. Medicine and Science in Sports and Exercise, 43(7), 1360-1368. https://doi.org/ 10.1249/MSS.0b013e318206476e

U.S. Department of Health and Human Services. (2010). Strategies to Improve the Quality of Physical Education. https://www.cdc.gov/healthyschools/pecat/ quality_pe.pdf

Verstraete, S. J., Cardon, G. M., De Clercq, D. L., \& De Bourdeaudhuij, I. M. (2006). Increasing children's physical activity levels during recess periods in elementary schools: the effects of providing game equipment. European journal of public health, 16(4), 415-419. https:/ /doi.org/10.1093/eurpub/ckl008

Viciana, J., Mayorga-Vega, D., \& Martínez-Baena, A. (2016). Moderate-to-Vigorous Physical Activity Levels in Physical Education, School Recess, and After-School Time: Influence of Gender, Age, and Weight Status. Journal of Physical Activity and Health, 13(10), 11171123. https://doi.org/10.1123/jpah.2015-0537

Wang, H., Li, T., Siahpush, M., Chen, L. W., \& Huberty, J. (2017). Cost Effectiveness of Ready for Recess to Promote Physical Activity in Children. Journal of School Health, 87(4), 278-285. https://doi.org/10.1111/josh.12495

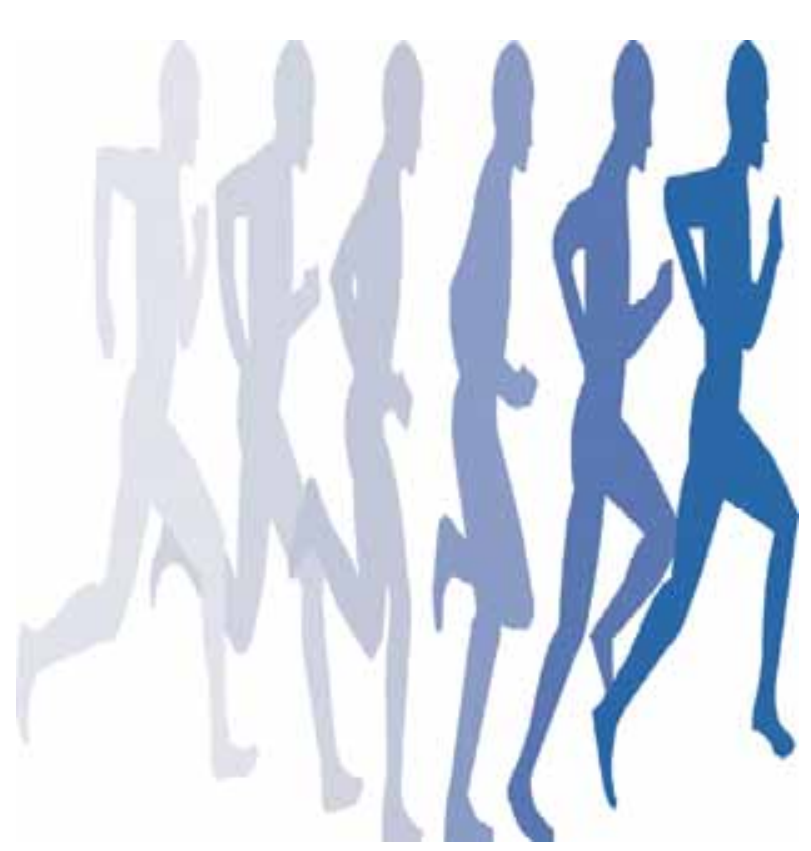

